\title{
Choice of Signaled or Unsignaled Onset of Differential Reinforcer Magnitudes
}

\author{
Ezra G. Hall \\ West Virginia University
}

Follow this and additional works at: https://researchrepository.wvu.edu/etd

\section{Recommended Citation}

Hall, Ezra G., "Choice of Signaled or Unsignaled Onset of Differential Reinforcer Magnitudes" (2012). Graduate Theses, Dissertations, and Problem Reports. 4861.

https://researchrepository.wvu.edu/etd/4861

This Thesis is protected by copyright and/or related rights. It has been brought to you by the The Research Repository @ WVU with permission from the rights-holder(s). You are free to use this Thesis in any way that is permitted by the copyright and related rights legislation that applies to your use. For other uses you must obtain permission from the rights-holder(s) directly, unless additional rights are indicated by a Creative Commons license in the record and/ or on the work itself. This Thesis has been accepted for inclusion in WVU Graduate Theses, Dissertations, and Problem Reports collection by an authorized administrator of The Research Repository @ WVU. For more information, please contact researchrepository@mail.wvu.edu. 
Choice of Signaled or Unsignaled

Onset of Differential Reinforcer Magnitudes

Ezra G. Hall

Thesis submitted to the Eberly College of Arts and Sciences at West Virginia University in partial fulfillment of the requirements for the degree of

Master of Science

in

Psychology

\author{
Kennon A. Lattal, Ph.D., Chair \\ Elizabeth G. E. Kyonka, Ph.D. \\ Miranda N. Reed, Ph.D. \\ Morgantown, West Virginia \\ 2012
}

Keywords: reinforcer magnitude; reinforcer onset; choice; post-reinforcement pause; pigeons 


\author{
ABSTRACT \\ Choice of Signaled or Unsignaled \\ Onset of Differential Reinforcer Magnitudes
}

Ezra G. Hall

In the most common operant procedure involving magnitude of reinforcement, single reinforcers, of one magnitude or the other, are available from the same source (with pigeons, a food hopper) at different times. The duration of access as a source of discriminative control by these reinforcers comes sometime after their onset, when one reinforcer continues for a longer duration than the other. Thus, reinforcers of different durations can be differentially reinforcing only after the passage of some time. In the current experiment, four pigeons responded on a single-key concurrent variable-time schedule of reinforcement. Two reinforcer durations, $2 \mathrm{~s}$ and $6 \mathrm{~s}$, were delivered within components of the concurrent schedule. This allowed covariation of magnitude within components while simultaneously covarying onset stimuli (red, green, and white hopper lights) between components. Time allocation to the schedule components did not vary as a function of differentially signaling reinforcer onset between components. Post-reinforcement pausing did vary as a function of the reinforcer duration: longer pausing occurred after 6-s reinforcers and shorter pausing occurred after 2-s reinforcers. These findings extend the generality of post-reinforcement pausing to variable-time schedules of reinforcement. 


\section{Acknowledgements}

I would like to thank Andy Lattal, Liz Kyonka, and Mirand Reed for serving as members of my thesis committee and for their helpful comments at all stages of this project. I would also like to thank Toshikazu Kuroda and August Holtyn for valuable conversations and help throughout the project. I am especially grateful to Andy Lattal for serving as my committee chair and advisor. 
Table of Contents

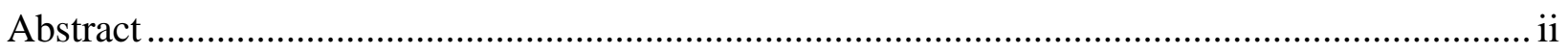

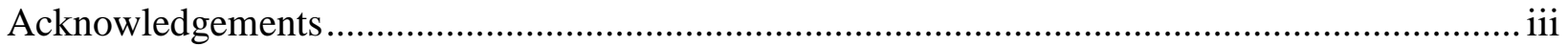

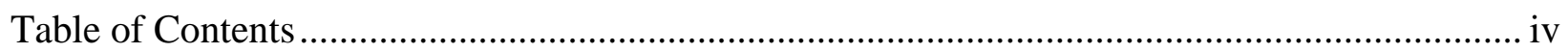

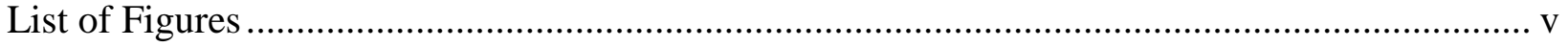

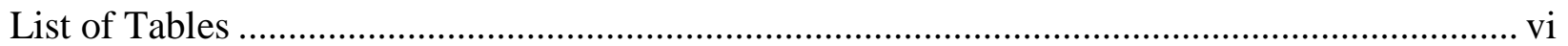

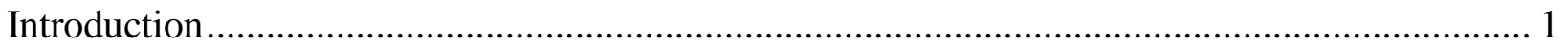

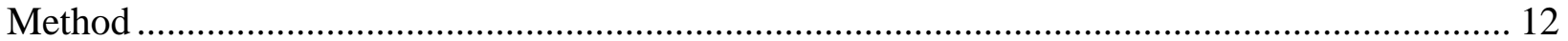

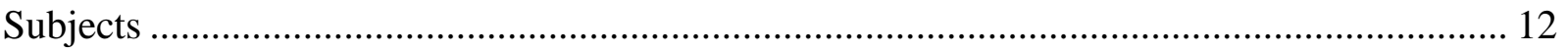

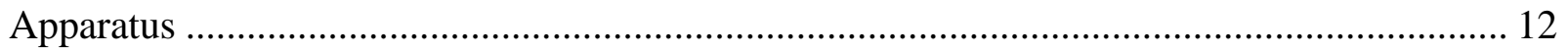

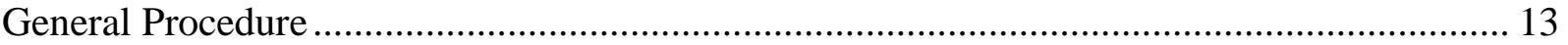

Interdependent Concurrent VT 120-s VT 120-s Schedule Phase ......................................... 14

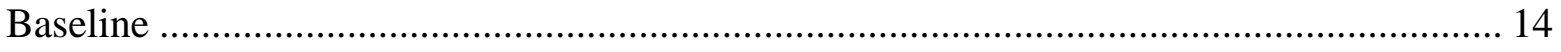

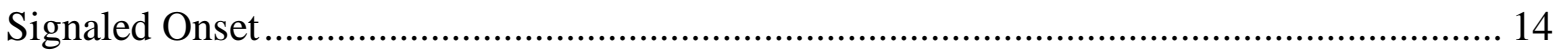

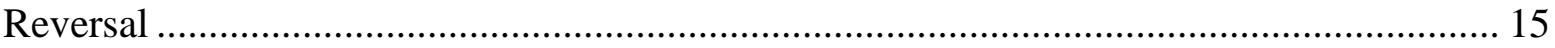

Independent Concurrent VT 120-s 120-s Schedule Phase .................................................. 15

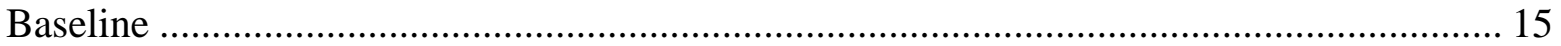

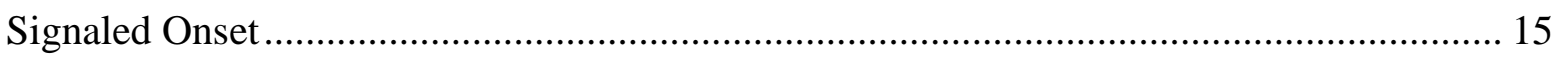

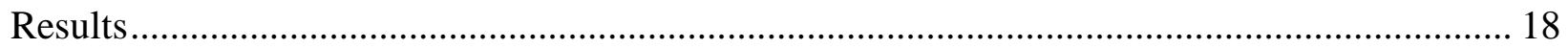

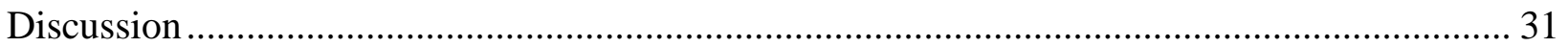

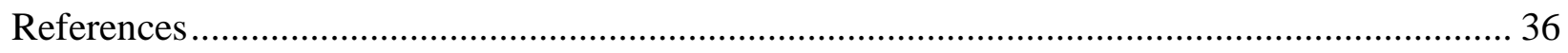




\section{List of Figures}

Figure 1. Mean proportions of session time calculated for one component from the last 6 stable sessions of each condition in Phase $1 . \mathrm{B}=$ Baseline, $\mathrm{S}=$ Signaled Onset, $\mathrm{R}=$ Reversal. The proportions for the signaled onset and reversal conditions are from the signaled component within each condition. The baseline proportions correspond to the same component represented in the signaled onset proportion. Error bars represent the standard deviation of the mean 20

Figure 2. Latency to a changeover response following long and short reinforcer durations in both components for each condition of Phase 1. B = Baseline, $\mathrm{S}=$ Signaled Onset, $\mathrm{R}=$ Reversal. Within each condition, the leftmost pair corresponds to the left hopper and the rightmost pair corresponds to the right hopper. Symbols represent medians and the bars represent the interquartile ranges $\left(25^{\text {th }}\right.$ to $75^{\text {th }}$ percentiles) of the last 6 stable sessions from each condition

Figure 3. Mean proportions of session time calculated for one component from the last 6 stable sessions of each condition in Phase 2. B = Baseline, $\mathrm{S}=$ Signaled Onset. The baseline proportions correspond to the same component represented in the signaled onset proportion. Error bars represent the standard deviation of the mean.

Figure 4. Latency to a changeover response following long and short reinforcer durations in both components for each condition of Phase 2. B = Baseline, $\mathrm{S}=$ Signaled Onset. Within each condition, the leftmost pair corresponds to the left hopper and the rightmost pair corresponds to the right hopper. Symbols represent medians and the bars represent the interquartile ranges $\left(25^{\text {th }}\right.$ to $75^{\text {th }}$ percentiles) of the last 6 stable sessions from each condition

Figure 5. Total changeover responses within each session for each condition of Phase 1 and Phase 2 starting with the last 6 stable sessions of the first baseline. Int $=$ Interdependent, Ind $=$ Independent, $\mathrm{B}=$ Baseline, $\mathrm{S}=$ Signaled Onset, $\mathrm{R}=$ Reversal 


\section{List of Tables}

Table 1. Magnitude and Correlated Hopper Stimuli and Number of Sessions for Each Condition of the Interdependent Concurrent VT 120 s VT 120 s Schedule.....

Table 2. Magnitude and Correlated Hopper Stimuli and Number of Sessions for Each Condition of the Independent Concurrent VT 120 s VT 120 s Schedule

Table 3. Time Allocation (s) from the Last 6 Stable Sessions for Each Condition of the Interdependent Concurrent VT 120 s VT 120 s Schedule

Table 4. Time Allocation (s) from the Last 6 Stable Sessions for Each Condition of the Independent Concurrent VT 120 s VT 120 s Schedule

Table 5. Mean Reinforcement Rate in Hopper Presentations Per Minute for the Last 6 Stable Sessions of the Interdependent Concurrent VT 120 s VT 120 s Schedule

Table 6. Mean Reinforcement Rate in Hopper Presentations Per Minute for the Last 6 Stable Sessions of the Independent Concurrent VT 120 s VT 120 s Schedule 
Introduction

Reinforcers can be arranged under a variety of schedules (e.g., fixed or variable ratio, or interval), configured in different ways (single, concurrent, multiple, mixed, etc.) and with alterations in parameters such as rate and delay to their delivery. Another variable that may affect behavior is the magnitude of the reinforcer delivered. Restricting the discussion to consumable reinforcers, some parameters of magnitude that can be manipulated include quality, concentration, volume, and the duration of access.

Changes in quality have been examined by providing access to high and/or low-preferred reinforcers within a session (cf. Ettinger, McSweeney, Norman, 1981). Concentration can be manipulated using solutions of sucrose or sweetened condensed milk by changing the ratio of water added to either (Baron, Mikorski, Schlund, 1992; Heyman \& Monaghan, 1994). Volume can be defined as the total amount of a reinforcer that is delivered and consumed (Keesey \& Kling, 1961; Young, 1981). Duration of access to a reinforcer is the amount of time programmed from its onset to offset and was described by Bonem and Crossman (1988) as the most commonly studied method of magnitude manipulation. When reinforcers of differing quality, concentration, or volume are delivered, they are immediately discriminable in their onset by sight or taste. Reinforcers of different durations of access are discriminable, or differentially reinforcing, only after the passage of some time. For example, $3 \mathrm{~s}$ and $6 \mathrm{~s}$ access to a reinforcer differentially reinforce behavior after 3 s has elapsed during the larger reinforcer delivery. This suggests two possible sources of control over behavior when a reinforcer is delivered: its onset and duration.

Given different durations of access, the most immediate effect of a reinforcer is its onset. If onset is undifferentiated between reinforcers (e.g. hopper presentations of mixed grain to 
pigeons as subjects), unlike comparisons of different qualities, concentrations, or volumes, different durations are not immediately discriminable as described above. It may be possible, however, to enhance discrimination of durations, and therefore their differential effectiveness, by signaling the onset of differential reinforcer magnitudes. The focus of the following experiment was an examination of signaling differential reinforcers magnitudes as duration of access. Conditions under which different reinforcer magnitudes defined as duration of access do and do not affect behavior first will be reviewed. The discriminability of reinforcement magnitude then will be discussed and extended to the examination of signaled and unsignaled onset of differential reinforcer magnitudes using a change-over operandum choice procedure (Findley, 1958).

Parameters of reinforcer magnitude other than duration

Concentration can be altered within or across sessions to examine the relative contributions of duration of access, percentage concentration, and any possible interactions between the two variables (Baron, Mikorski, Schlund, 1992; Heyman \& Monaghan, 1994). Defining the quality of a reinforcer is difficult because quality can depend on various establishing operations and other factors such as nutritional value (given a current state) or preference of one reinforcer over another (e.g. Ettinger, McSweeney, Norman, 1981). The volume of a reinforcer can change the duration of consummatory response thereby increasing the total duration of access. Keesey and Kling (1961) addressed the duration of the consummatory response by equating the number of dried peas presented per reinforcer delivery while altering the overall volume. They examined the effects of different volumes of dried peas on key-peck responding of pigeons maintained by variable-interval (VI) schedules of reinforcement. They divided the peas so that the number of pieces per reinforcer delivery was always 4 , although the 
total volume to be consumed was different (1, 2, 3, and 4 peas). Daily alternations of baseline (quarter pea reinforcer only) and one of the four experimental amounts occurred, each paired with a different key color, until responding stabilized to each of the four experimental amounts. Orderly changes in stable response rates were not obtained as a function of the volume of reinforcement delivered. Following stability at the baseline amount, five probe stimuli previously correlated with the experimental amount were introduced for $90 \mathrm{~s}$ during sessions. Differential response rates, seen only at the beginning of the sessions, occurred in the presence of the probe stimuli. That is, response rates varied as a function of the previous stimulus and reinforcer pairing. When the reinforcer number was not equated (using 2, 4, and 8 hemp seeds per reinforcement in experiment II) similar results were obtained. Although concentration, quality, and volume all warrant further examination, duration of access to mixed grain with pigeons as subjects will comprise the remaining discussion of reinforcer magnitude.

\section{Duration of reinforcer access: effects of single key schedules}

Catania (1963) examined single and two-key arrangements for delivering differential reinforcer magnitudes. Key pecking of pigeons was maintained by variable interval (VI) 2-min schedules of food reinforcement. Three reinforcer durations were examined across sessions (3 s, $4.5 \mathrm{~s}$, and $6 \mathrm{~s}$ ) using a single key. The single-key procedure produced unsystematic changes in response rates among the three magnitudes. In the two-key (concurrent schedule) procedure, two durations were assigned separately to each key and were held constant across sessions in a condition. Catania obtained a linear relation between responses per min and duration of reinforcer presentation. This result suggests that the context of the second key provided a means of discrimination of the differential reinforcer durations within sessions. This also demonstrates that single key schedules of reinforcement can be relatively insensitive to changes in reinforcer 
magnitude across sessions and is similar to the finding of Keesey and Kling (1961) where rate of responding was insensitive to maintained magnitude conditions.

Stimulus conditions and the discrimination of duration: multiple and concurrent schedules

The following conditions are not exhaustive of those that can accompany changes in reinforcer magnitude, but are examples of covariation of magnitude and stimulus conditions (as per Bonem and Crossman, 1988) that may be responsible for magnitude discrimination and the appearance of systematic effects given a variety of schedule types and configurations as presented in the introduction: the specific key (left, center, right), key light color, and conditions accompanying the onset of the reinforcers. Multiple and concurrent chain procedures will be reviewed here followed by a discussion of observing stimuli and differential hopper stimuli in the next section.

Multiple schedules (mult) allow different magnitudes to be correlated with different stimuli using a single key. Unlike Keesey and Kling (1961), where the stimuli and associated magnitudes were alternated in separate daily sessions (with later additions of nonreinforced probe stimuli correlated with previous magnitudes), conventional multiple schedules examine within session stimulus alternations. For example, Shettleworth and Nevin (1965) examined response rate using a mult VI 2-min VI 2-min schedule of reinforcement where red and green stimuli alternated every $3 \mathrm{~min}$. Relative response rate varied as a function of the relative reinforcement duration within each component across conditions; the larger magnitude component showing higher relative response rates.

An experiment by Neuringer (1967) extended the analysis of single and two-key magnitude manipulations by restricting responding to one of two alternatives after a choice response was made. Neuringer arranged a concurrent-chain where an initial choice (FR1) of 
either the left or right key (both lighted white) initiated a 5-s timer and simultaneously darkened the opposite key. The chosen key remained white. After the 5-s timer elapsed, two scenarios could occur: 1) If a reinforcer was set up by one of the two independently timed and concurrently active VI 60-s schedules on the chosen key, it could be earned by the first response after $5 \mathrm{~s}$, followed in turn by a new choice trial, or 2) if a reinforcer was not set up there, a 1-s blackout started immediately after $5 \mathrm{~s}$ followed by a new choice trial. One initial link key led to a constant-duration (2 s) reinforcer while the other led to varied durations (2 s - $10 \mathrm{~s}$ ) across conditions. Choice proportion favored the key leading to the larger reinforcer duration across conditions; however, Neuringer did not find systematic changes in response rate during the $5 \mathrm{~s}$ periods that culminated with the larger reinforcer magnitudes.

Two methodological considerations may help explain why response rate did not increase during the $5 \mathrm{~s}$ leading to the larger reinforcer magnitudes. First, although the two keys were correlated with differential magnitudes, a choice of either key did not necessarily lead to reinforcement at the end of each trial. A stimulus change during the 5-s delay signaling the upcoming reinforcer magnitude could have enhanced response rates to the higher magnitude component. Second, we can assume that the pigeons were spending relatively more time in the larger magnitude component (as evidenced by the increase in choice proportion there), thus allowing the probability of a reinforcer delivery in the constant component to increase, thereby decreasing the probability of experiencing a blackout. Neuringer discussed this issue and suggested that choice and response rates in this experiment were controlled by different dimensions of the reinforcing stimulus respectively: the magnitude and the probability of occurrence. 
The procedure used by Neuringer (1967) can be conceptualized as a mixed concurrent chain because a choice response did not lead to any differential post-choice stimulus, but rather the activation of the programmed contingency (terminal links). Therefore the key position likely acted as a discriminative stimulus for the upcoming reinforcer magnitude. Concurrent-chains schedule provide the opportunity to vary multiple stimulus conditions correlated with differential reinforcer magnitudes within and across sessions. Such an examination was carried out by Ploog (2001) by comparing differential and nondifferential terminal link stimuli. The left and right key initial link stimuli always were correlated with differential reinforcer magnitudes in their respective terminal links (Condition 1: $3 \mathrm{~s}$ vs. $6 \mathrm{~s}$; Condition 2: $1 \mathrm{~s}$ vs. $6 \mathrm{~s}$ ) although their position alternated within a session. Terminal links could be entered after satisfying independently timed VI 60-s schedules. The terminal link always was associated with the center key, but one group of pigeons received differential stimuli correlated with the two reinforcer durations and one group received nondifferential stimuli. Initial link choice was indifferent at $3 \mathrm{~s}$ vs. $6 \mathrm{~s}$ and slightly favored the larger magnitude at $1 \mathrm{~s}$ vs. $6 \mathrm{~s}$ (.05 above indifference) when the terminal links were non differential, but was almost exclusive for the larger reinforcer duration in the 1-s vs. 6-s condition when the terminal link stimuli were differential. This highlights the importance of discrimination between reinforcer durations.

If there was evidence of control by the larger reinforcer duration with nondifferential stimuli, the choice proportion leading to that larger alternative would have been greater than approximately .50 seen in Ploog (2001). Differentially signaling the upcoming reinforcer duration allowed magnitude discrimination to occur. Perone and Courtney (1992) examined fixed-ratio pausing as effects of reinforcer magnitude manipulations in single-key mixed and multiple schedules. Although the dependent measure is different than that used by Ploog, they 
also found that the stimulus conditions correlated with the upcoming magnitude exerted discriminative control. When a mixed schedule was in effect, pausing varied as a function of the past reinforcer, with larger pauses following larger reinforcer magnitudes. When a multiple schedule was in effect, pauses were longer when the upcoming reinforcer was small and longer pauses still occurred after the larger reinforcer as in the mixed schedule.

Stimulus conditions and discrimination of duration: observing stimuli and hopper stimuli

Observing responses are used to change a mixed schedule to a multiple schedule (Wyckoff, 1952). Responding does not alter the schedule of reinforcement in effect, but rather the stimulus condition under which responding occurs. Auge (1973) used this procedure to signal the magnitude of the upcoming reinforcer. Responses to an observing key were maintained when they resulted in stimulus changes to the food key, presented until reinforcement, which signaled differential reinforcer magnitudes (10-s or 2-s access to mixed grain). Observing was not maintained, however, when only brief stimulus presentations signaled both magnitudes and when only the smaller reinforcer magnitude was signaled. This suggests that subjects will respond to a key that provides a signal of the upcoming reinforcer duration given that the signal is of a sufficient duration and occasions a larger (or does not occasion a smaller) reinforcer magnitude.

The procedures discussed so far have described conditions where an effect of magnitude is seen and those where such an effect is not seen. The stimulus conditions accompanying magnitude changes in the experiments up to this point in the discussion have not been contiguous with the onset of a reinforcer. The onset of differential reinforcer magnitudes provides an additional context where stimulus discrimination is possible. Mariner and Thomas (1969) suggested that using the same stimulus (e.g., feeder light intensity) for two different durations of 
magnitudes produces delayed contact with those differential reinforcement durations. For example, when comparing 2-s and 6-s durations, at least $2 \mathrm{~s}$ must pass during a presentation of the 6-s reinforcer for the subject to be differentially affected by this duration. To test whether signaling the onset of differential reinforcers exerts control over behavior, Mariner and Thomas altered the intensity of the food magazine light accompanying 2-s and 6-s reinforcement durations for one group (the "cued" group) of pigeons and presented the same food magazine light intensity for both durations with another group (the "non-cued” group). A multiple schedule was used for both groups where key light color and reinforcer magnitude covaried between components. That is, key colors were correlated with one magnitude. Each component was separated from the next by a 10-s timeout. The main purpose of their experiment was to examine peak shift in post discrimination gradients, but the present concern is with the initial discrimination training results of the two groups. Response rates for the "cued" group showed greater differentiation between the two components (and thus reinforcer magnitudes) of the multiple schedule than those of the "non-cued" group. These findings, although limited in that the conditions were not run to stability, suggest an effect of stimuli correlated with reinforcer duration and provide an alternative context where reinforcer discrimination can be manipulated. Reinforcer magnitude effects using the Findley (1958) procedure

Catania’s (1963) simultaneous presentation of different magnitudes on two keys provided a method that revealed behavioral sensitivity to changes in reinforcer magnitude. Previously, Findley (1958) designed a procedure where one stimulus was present at a time on one key and a change to another stimulus on that key could be made by responding on a second changeover key. This provided an explicit measure of changing over between two alternatives. Examinations using the two-key concurrent scheduling and the changeover-key design 
(Brownstein \& Pliskoff, 1968; Davison \& Hogsden, 1984; Davison, 1988) with outcome measures such as time allocation, rather than response rate, have been shown to be proportional to the rate of reinforcement provided in each alternative (Baum \& Rachlin, 1969). Given the consistency between results of these different concurrent scheduling methods and outcome measures, one way that the change-over key design has been uniquely used is in the study of response-independent reinforcers on choice. It would not be possible to discriminate between key sides, color, and any corresponding reinforcer magnitude changes using responseindependent reinforcer deliveries with a two-key concurrent schedule.

Behavior maintained by response-independent reinforcers delivered under variable-time (VT) schedules of reinforcement arranged concurrently using a changeover key procedure is sensitive to changes in reinforcer magnitude (Brownstein, 1971). Relative time allocation in both components was shown to approximate the reinforcer duration presented there. The changeover-key procedure delivering response independent reinforcers is a good method to examine signaling the onset of differential reinforcer magnitudes because of the precise measure of choice as time allocation between two alternatives and because the response-independent reinforcer deliveries allow attending to the hopper stimulus when a reinforcer occurs rather than pecking a key at the moment of reinforcer delivery. The changeover key and responseindependent reinforcer delivery combination seems ideal to maximize any functional change in choice between two alternatives: one providing signaled reinforcer onset, the other providing unsignaled reinforcer onset.

\section{Statement of the Problem}

In the most common operant procedure involving magnitude of reinforcement, single reinforcers, of one magnitude or the other, are available from the same source (with pigeons, a 
food hopper) at different times. The duration of access as a source of discriminative control by these reinforcers comes sometime after their onset, when one reinforcer continues for a longer duration than the other. Thus, reinforcers of different durations can be differentially reinforcing only after the passage of some time. This leaves reinforcer onset as the most immediate effect. The research reviewed here and more extensively elsewhere (see Bonem \& Crossman, 1988) have shown mixed effects of such reinforcer magnitude on operant behavior. A key feature where effects of reinforcer magnitude, defined as duration of access, are seen is whether there is a covariation of the two magnitudes and stimulus conditions. That is, differential reinforcement of behavior is enhanced when different durations of access are correlated with distinct stimuli. When, for example, Catania (1963) varied duration of access across sessions using a single key, there was no systematic effect of magnitude. It was not until the different durations were presented following responding on two distinct keys within a single session that a systematic increase in response rate to the larger reinforcer magnitude occurred. That is, magnitude had an effect when one duration was available for immediate (concurrent) contrast with the other duration. Therefore, correlation of magnitudes with the left and right keys allowed discriminative control to occur.

Similar differential effects of magnitude under stimulus control have been reported in multiple schedules (Shettleworth \&Nevin, 1965) and in an observing response procedure when two different magnitudes were associated with different stimuli in an observing response procedure (Auge, 1973). Furthermore, when the terminal links of concurrent chains signal differential reinforcer durations, choice proportions favor the larger duration. When the terminal links provide no stimulus differentiation between reinforcer durations, discriminability may be degraded and the choice proportion tends toward indifference (Ploog, 2001). Ploog’s data 
suggest that stimuli accompanying the onset of different reinforcer magnitudes lead to differential control of responding by reinforcer magnitude.

The only experiment attempting a direct assessment of the relative roles of reinforcer onset and subsequent duration of reinforcer access in controlling responding was conducted by Mariner and Thomas (1969). They correlated bright and dim hopper lights with different durations of access presented in alternating components of a multiple schedule. Increased differentiation in response rate between components occurred when large and small durations had differentially signaled onsets compared to when the onset stimuli were not differential between the durations. These results suggest that the control of behavior by reinforcer magnitude may be in part a function of the discriminability of the reinforcer at its onset.

Comparisons of signaled and unsignaled onset of differential reinforcer magnitudes merit further investigation to determine if extended functional control of behavior by reinforcement magnitude is possible through their manipulation. If covariation of stimulus conditions contiguous with the onset of differential reinforcer magnitudes differentially affects behavior then this would confirm two different sources of control when a reinforcer is delivered: its onset and duration. Mariner and Thomas's (1969) experiment suggest such a source of control, but the absence of stability criteria and the limited number of sessions conducted restrict the conclusions that can be drawn from their data. The current experiment therefore arranged two reinforcer durations to be delivered within components of a concurrent schedule. This allowed covariation of magnitude within components while simultaneously covarying onset stimuli between components. 


\section{Method}

Subjects

Four White Carneau pigeons with a history of responding under schedules of positive reinforcement were used. Each was housed individually with continuous access to water in a vivarium with a 12:12 hr light/dark cycle. They were maintained at $80 \%$ of their ad libitum body weight (+/- 15 g) by post-session feedings that occurred at least 30 minutes after sessions. Apparatus

One operant conditioning chamber for pigeons with a work area $31 \mathrm{~cm}$ long by $31 \mathrm{~cm}$ wide by $39 \mathrm{~cm}$ high was used. One wall of the work area had an aluminum work panel containing one 2-cm diameter response key located $13 \mathrm{~cm}$ (this and all other measurements are center to center) from the top and bisecting the midline of the work panel. It was operated by a minimum force of .15 N. Blue and orange filtered 28-vdc bulbs were located behind the response key. A house light was located behind a $4 \mathrm{~cm}$ by $4 \mathrm{~cm}$ opening covered with translucent plastic on the midline of the work panel $4 \mathrm{~cm}$ from the floor. Reinforcement was Purina Nutri Blend pigeon pellets available from two hoppers (Lehigh Valley Electronics Lshaped with top pivot point) located behind two $5 \mathrm{~cm}$ by $6 \mathrm{~cm}$ apertures $9 \mathrm{~cm}$ from the floor and $8 \mathrm{~cm}$ on either side of the midline. When the left and right hopper were activated it was possible for them to be illuminated by either a $28 \mathrm{v}$ red, green or white light located inside and above the aperture. The orange and blue key light colors were correlated with either the left or the right hopper. A ventilation fan and white noise masked extraneous sound. A computer located outside the experimental room ran MedPC 7 software controlled the experimental procedures and recorded the data. 


\section{General Procedure}

Preliminary training consisted of leaning the mean interreinforcer interval over the course of approximately 20 days until the terminal value of $60 \mathrm{~s}$ was reached for each pigeon.

Subsequently, each session began with a 180-s blackout in the chamber, during which key and houselights were turned off. After the blackout, one of two key-light colors (blue or orange) was turned on with a .5 probability. Each key light color was associated with one component of a concurrent VT 120-s VT 120-s schedule and was correlated with food presentation from either the left or right hopper, counterbalanced across pigeons, for all conditions. A single response to the key changed the key color (c.f. Findley, 1958) and started a 3-s changeover delay (COD; Herrnstein, 1961), ensuring that a reinforcer delivery occurred within at least 3 s from a changeover response. Reinforcers in the two components were delivered independently of responding from the two food hoppers (hereafter, “left and right hoppers”). Short (2 s) and long (6 s) hopper durations within either component occurred quasi randomly, with the restriction of no more than 4 consecutive reinforcers of the same magnitude occurring from the same hopper. During reinforcement, the appropriate hopper light was turned on and the house and key lights were turned off. In baseline conditions, white hopper lights accompanied all reinforcer deliveries from the left and right hopper. In the signaled onset and reversal conditions, both short and long reinforcers delivered in one component always were associated with white hopper lights while in the other component red and green hopper lights were associated with either the short or long duration.

The experiment was comprised of two phases: one using an interdependent concurrent schedule and the other an independent concurrent schedule. The sequence of conditions within each phase and number of sessions at each condition are shown in Tables 1 and 2. The 
conditions were in effect for a minimum of 20 sessions and until time allocation in each component differed by no more than \pm .05 of the mean proportion of the last six consecutive sessions. With rare exception, sessions occurred seven days a week at approximately the same time each day and ended after the delivery of 48 reinforcers.

Interdependent Concurrent VT 120-s VT 120-s Schedule Phase.

During each condition in this phase, successive interreinforcer intervals were drawn without replacement from a single distribution of 12 intervals derived from the distribution described by Fleshler \& Hoffman (1962). The two components were interdependent because a reinforcer had to be delivered before the next VT value started to time down (Stubbs \& Pliskoff, 1969). The reinforcers were assigned quasi-randomly to either key color. Reinforcers were delivered from their appropriate hopper with the restriction of no more than 3 consecutive presentations in a row from the same hopper. Twelve 6-s and twelve 2-s reinforcers were delivered to the left and right hopper within a session. Within this phase, the following conditions remained in effect until time allocation stabilized:

Baseline. A white hopper light was used for all reinforcer deliveries from both hoppers in this condition.

Signaled Onset. This condition was identical to the baseline, except for the addition of red and green hopper lights signaling the onset of the differential magnitudes in one of the hoppers (signaled onset component). All of the unsignaled hopper reinforcers had a white hopper light (unsignaled onset component). Table 1 shows the hopper light configurations for each pigeon. 
Reversal. This condition was identical to the previous condition except that the hopper associated with white or red and green hopper lights was reversed. The key light color and hopper correlation remained the same.

Independent Concurrent VT 120-s 120-s Schedule Phase.

The concurrent schedule was identical to that described in the Interdependent Concurrent VT 120-s VT 120-s Schedule Phase except that intervals simultaneously and independently timed down. This arrangement allowed the total number of short and long reinforcers to vary within a component depending on the total time allocation within each session. Within this phase, the following conditions remained in effect until responding stabilized:

Baseline. A white hopper light was used for all reinforcer deliveries from both hoppers in this condition.

Signaled Onset. This condition was similar to the signaled onset condition mentioned in Interdependent Condurrent VT 120-s 120-s Schedule Phase. Table 2 shows the hopper light configurations for each pigeon within this condition. 
Table 1

Magnitude and Correlated Hopper Stimuli and Number of Sessions for Each Condition of the Interdependent Concurrent VT 120 s VT 120 s Schedule

\begin{tabular}{llccc} 
Pigeon & Condition & Left Hopper & Right Hopper & Sessions \\
\hline \hline 828 & Baseline 1 & & 55
\end{tabular}

Signaled Onset 6-s white / 2-s white 6-s red / 2-s green 22

Reversal 6-s red / 2-s green 6-s white / 2-s white 26

Baseline $2 \quad 28$

$806 \quad$ Baseline $1 \quad 62$

Signaled Onset 6-s green / 2-s red 6-s white / 2-s white 34

Reversal 6-s white / 2-s white 6-s green / 2-s red 20

Baseline 2 20

$\begin{array}{lll}56 & \text { Baseline } 1 & 61\end{array}$

Signaled Onset 6-s white / 2-s white 6-s red / 2-s green 21

Reversal 6-s red / 2-s green 6-s white / 2-s white 27

Baseline 2

2 Baseline $1 \quad 85$

Signaled Onset 6-s white / 2-s white 6-s green / 2-s red 24

Reversal 6-s green / 2-s red 6-s white / 2-s white 26

Baseline $2 \quad 26$

Note. Baseline conditions have all white hopper lights. Pigeons 828 and 806 had the blue key light correlated with the left hopper and the orange key light correlated with the right hopper. Pigeons 56 and 2 had the key light and hopper correlations reversed. 
Table 2

Magnitude and Correlated Hopper Stimuli and Number of Sessions for Each Condition of the Independent Concurrent VT 120 s VT 120 s Schedule

\begin{tabular}{|c|c|c|c|c|}
\hline Pigeon & Condition & Left Hopper & Right Hopper & Sessions \\
\hline 828 & Baseline 1 & & & 21 \\
\hline
\end{tabular}

Signaled Onset 6-s white / 2-s white 6-s green / 2-s red 27

\begin{tabular}{lr} 
Baseline 2 & 21 \\
\hline
\end{tabular}

$\begin{array}{lll}806 & \text { Baseline } 1 & 20\end{array}$

Signaled Onset 6-s red / 2-s green $\quad$ 6-s white / 2-s white 21

\begin{tabular}{lr} 
Baseline 2 & 20 \\
\hline
\end{tabular}

$56 \quad$ Baseline $1 \quad 23$

Signaled Onset 6-s white / 2-s white 6-s green / 2-s red 20

\begin{tabular}{lr} 
Baseline 2 & 20 \\
\hline
\end{tabular}

$2 \quad$ Baseline $1 \quad 36$

Signaled Onset 6-s white / 2-s white 6-s red / 2-s green 20

Baseline 2

Note. Baseline conditions have all white hopper lights. Pigeons 828 and 806 had the blue key light correlated with the left hopper and the orange key light correlated with the right hopper. Pigeons 56 and 2 had the key light and hopper correlations reversed. 


\section{Results}

Figure 1 shows mean proportion of session time spent in the presence of one of the hopper components during the last 6 (stable) sessions for each condition of Phase 1 . The proportions for the signaled onset and reversal conditions are from the signaled onset component. The baseline proportions displayed are from the same hopper component as presented in the signaled onset condition (see Table 1 and Figure 1 caption for a detailed description). The component times used to generate the proportions are shown in Table 3. Mean proportion of session time in the signaled component for Pigeons 828, 806, and 2 was not systematically higher than the unsignaled component within each condition or the corresponding component in the first baseline condition. For Pigeon 56, the mean proportion of session time in the signaled component during the reversal condition was higher than the unsignaled component within this condition, the signaled component in the preceding signaled onset condition, and both baseline conditions.

Figure 2 shows the median latencies to a changeover response following long and short reinforcer deliveries for the last 6 (stable) sessions for both components of all conditions in Phase 1. Latencies were determined by recording the time from each reinforcer offset until the next response occurred. The times between two consecutive reinforcer deliveries without a changeover response were excluded from the analysis. The latencies show differences in the post-reinforcement pause following reinforcer deliveries within each component of each condition. For all pigeons, excluding the signaled component in the reversal condition for Pigeon 2, the median latencies were longer following 6-s reinforcers than 2-s reinforcers. For Pigeons 828 and 806, the latencies for both 6-s and 2-s reinforcers in the signaled component were shorter when compared to the corresponding component in the preceding baseline and also 
shorter than the corresponding magnitude of reinforcement in the unsignaled component within this condition. The reversal condition for Pigeon 56 shows longer latencies for the signaled component than the unsignaled component, but the latencies remained similar to those during the preceding signaled onset condition. 


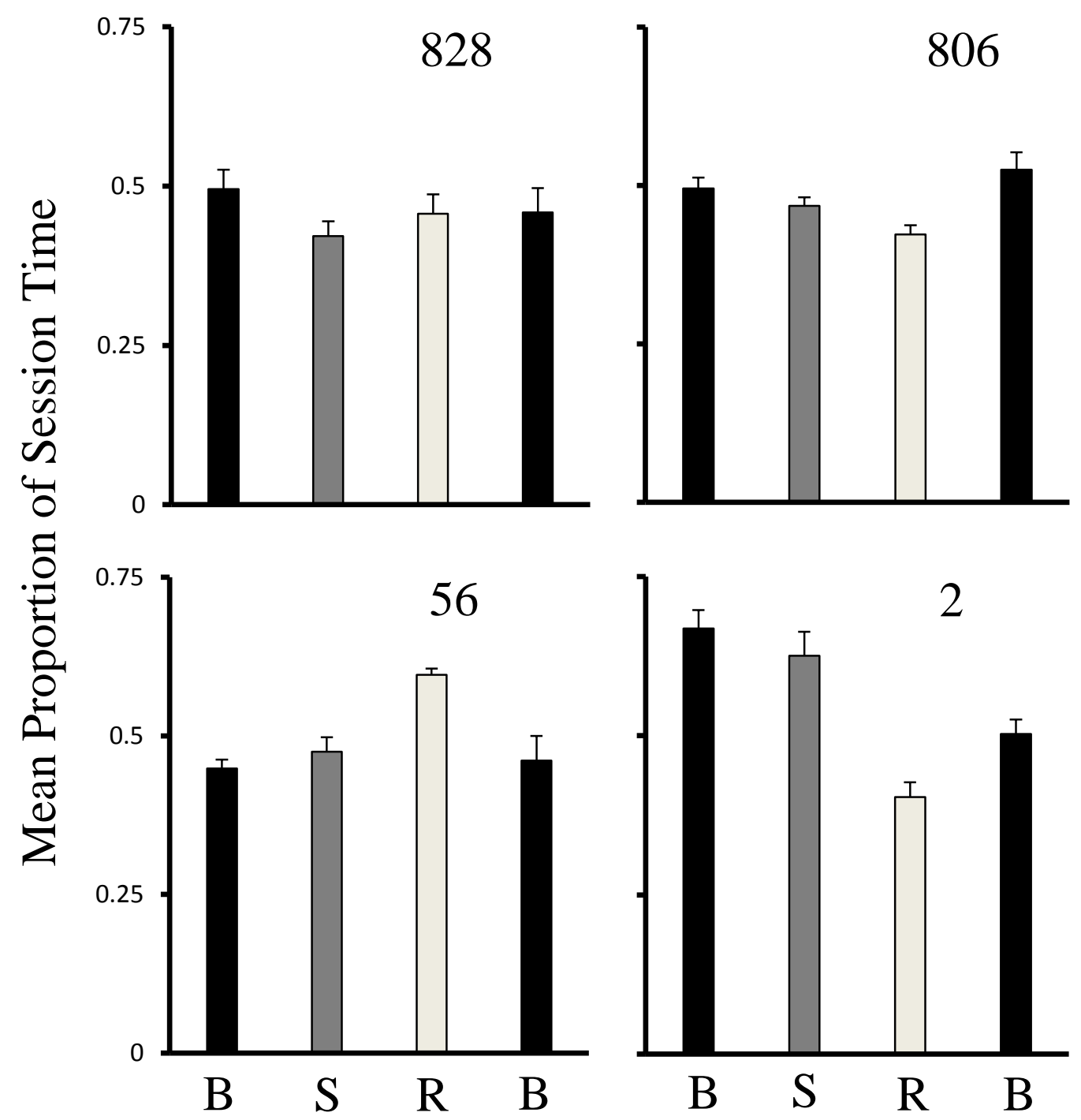

Figure 1. Mean proportions of session time calculated for one component from the last 6 stable sessions of each condition in Phase $1 . \mathrm{B}=$ Baseline, $\mathrm{S}=$ Signaled Onset, $\mathrm{R}=$ Reversal. The proportions for the signaled onset and reversal conditions are from the signaled component within each condition. The baseline proportions correspond to the same component represented in the signaled onset proportion. Error bars represent the standard deviation of the mean. 
Table 3

Time Allocation (s) from the Last 6 Stable Sessions for Each Condition of the Interdependent Concurrent VT 120 s VT 120 s Schedule

\begin{tabular}{llllllll}
\multicolumn{2}{c}{ Baseline 1 } & \multicolumn{2}{c}{ Signaled Onset } & \multicolumn{2}{c}{ Reversal } & \multicolumn{2}{c}{ Baseline 2 } \\
\hline Left & Right & Left & Right & Left & Right & Left & Right \\
\hline \hline
\end{tabular}

Pigeon 828

$\begin{array}{llllllll}1885.96 & 1534.77 & 1778.53 & 1442.25 & 1388.65 & 1896.75 & 1723.51 & 1516.19 \\ 1664.94 & 1741.62 & 1916.66 & 1401.64 & 1388.87 & 1807.04 & 1711.90 & 1440.75 \\ 1521.43 & 1791.15 & 1996.15 & 1281.95 & 1573.97 & 1533.91 & 1879.42 & 1366.47 \\ 1745.63 & 1722.74 & 1958.59 & 1302.67 & 1552.05 & 1701.11 & 1809.43 & 1334.72 \\ 1695.82 & 1603.47 & 1869.63 & 1380.68 & 1518.61 & 1824.00 & 1731.19 & 1461.31 \\ 1650.10 & 1570.33 & 1856.47 & 1483.33 & 1473.62 & 1839.28 & 1580.18 & 1748.90\end{array}$

Pigeon 806

$\begin{array}{llllllll}1631.79 & 1497.45 & 1453.06 & 1694.08 & 1759.78 & 1424.52 & 1686.18 & 1463.16 \\ 1617.74 & 1570.59 & 1467.82 & 1657.70 & 1819.40 & 1295.55 & 1521.58 & 1618.93 \\ 1525.33 & 1696.60 & 1552.43 & 1624.36 & 1822.33 & 1277.17 & 1647.88 & 1507.54 \\ 1512.74 & 1591.80 & 1449.76 & 1631.53 & 1838.94 & 1405.15 & 1766.20 & 1340.27 \\ 1532.37 & 1633.58 & 1373.76 & 1703.44 & 1844.85 & 1280.03 & 1657.50 & 1532.07 \\ 1579.35 & 1588.46 & 1507.84 & 1701.08 & 1794.76 & 1294.81 & 1655.93 & 1523.10\end{array}$

Pigeon 56

$\begin{array}{llllllll}1796.31 & 1408.17 & 1704.17 & 1497.65 & 1889.95 & 1322.55 & 1847.80 & 1317.46 \\ 1715.14 & 1502.51 & 1725.58 & 1456.42 & 1899.04 & 1342.30 & 1609.95 & 1656.41 \\ 1804.68 & 1385.21 & 1573.11 & 1569.65 & 1955.19 & 1275.89 & 1775.56 & 1478.86 \\ 1736.58 & 1450.55 & 1598.84 & 1611.83 & 1890.56 & 1271.57 & 1609.74 & 1660.89 \\ 1857.97 & 1433.04 & 1803.21 & 1437.40 & 1859.94 & 1288.24 & 1773.82 & 1476.91 \\ 1681.00 & 1429.70 & 1646.87 & 1512.97 & 1944.81 & 1242.22 & 1971.32 & 1467.04\end{array}$

Pigeon 2

\begin{tabular}{llllllll}
1252.74 & 2042.21 & 1428.99 & 2104.71 & 1413.61 & 1889.96 & 1588.32 & 1766.95 \\
1111.33 & 2435.57 & 1054.98 & 2284.22 & 1372.51 & 1916.08 & 1545.74 & 1697.57 \\
1140.95 & 2517.91 & 1358.57 & 2111.87 & 1334.32 & 2073.77 & 1677.66 & 1493.51 \\
1209.42 & 2231.94 & 1389.68 & 1926.34 & 1254.37 & 2049.65 & 1576.94 & 1592.97 \\
1123.62 & 2332.57 & 1253.60 & 2225.98 & 1340.09 & 2193.40 & 1663.52 & 1534.49 \\
1067.58 & 2411.16 & 1147.49 & 2087.17 & 1423.47 & 1910.95 & 1605.94 & 1687.60 \\
\hline
\end{tabular}

Note. Left and Right designate hoppers. 

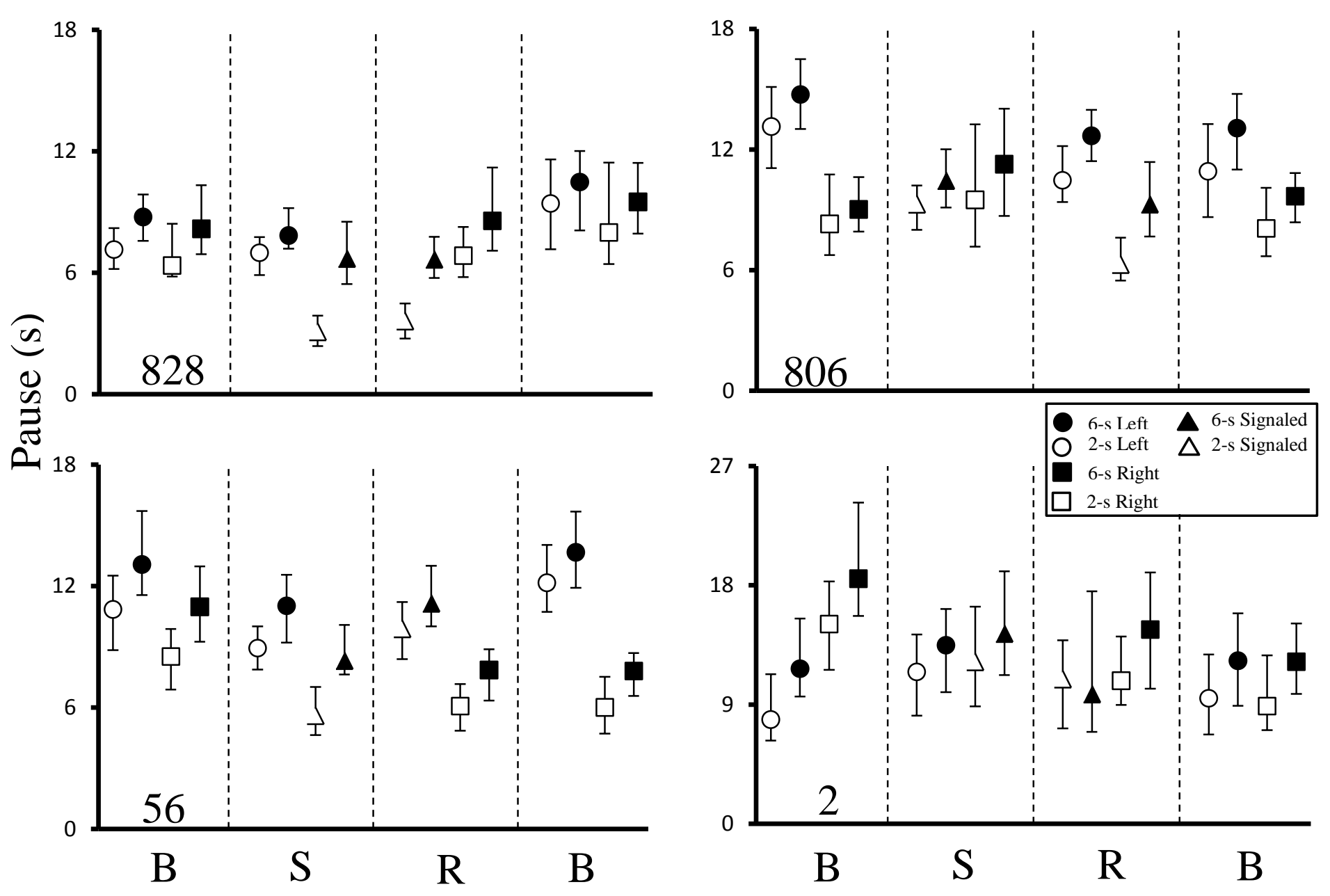

Figure 2. Latency to a changeover response following long and short reinforcer durations in both components for each condition of Phase 1. B = Baseline, $\mathrm{S}=$ Signaled Onset, $\mathrm{R}=$ Reversal. Within each condition, the leftmost pair corresponds to the left hopper and the rightmost pair corresponds to the right hopper. Symbols represent medians and the bars represent the interquartile ranges (25th to 75th percentiles) of the last 6 stable sessions from each condition. 
Figure 3 shows mean proportion of session time spent in the presence of one of the hopper components during the last 6 stable sessions for each condition of Phase 2. Each proportion corresponds to the same hopper component (see Table 2 and Figure 3 caption for a detailed description). The component times used to generate the proportions are shown in Table 4. As in the prior phase, the proportions did not vary systematically as a function of the signaled onset. The increased time allocation for Pigeon 56, seen in the reversal condition of Phase 1, was not replicated in this phase. That is, time allocation to the signaled onset component did not remain above $50 \%$ in the signaled onset component.

Figure 4 shows the median latencies to a changeover response following long and short reinforcer deliveries for the last six stable sessions for both components of all conditions in Phase 2. Similar to Phase 1, the median latencies in Phase 2 were generally longer following 6-s reinforcers than 2-s reinforcers. Two exceptions should be noted: the right hopper component for Pigeon 828 in the second baseline and the unsignaled component in the signaled onset condition for Pigeon 56 did not follow this pattern.

Figure 5 shows the total changeover responses during each session of every condition starting with the last 6 stable sessions of the first baseline condition. For Pigeons 828, 56, and 2 there was a decreasing trend in the number of changeover responses across all sessions. For Pigeon 806, there was an initial increase in changeover responses beginning with the first signaled onset condition and a subsequent decrease to initial baseline levels by the reversal condition. There were no other systematic increases or decreases between changeover responses within a condition or between conditions for any of the pigeons.

Reinforcement rate as hopper presentations per minute in the last 6 stable sessions for all conditions of Phase 1 and Phase 2 are shown in Tables 5 and 6, respectively. Hopper 
presentations per minute for all pigeons in Phase 1 (interdependent schedule) were slightly lower than in Phase 2 (independent schedule). 

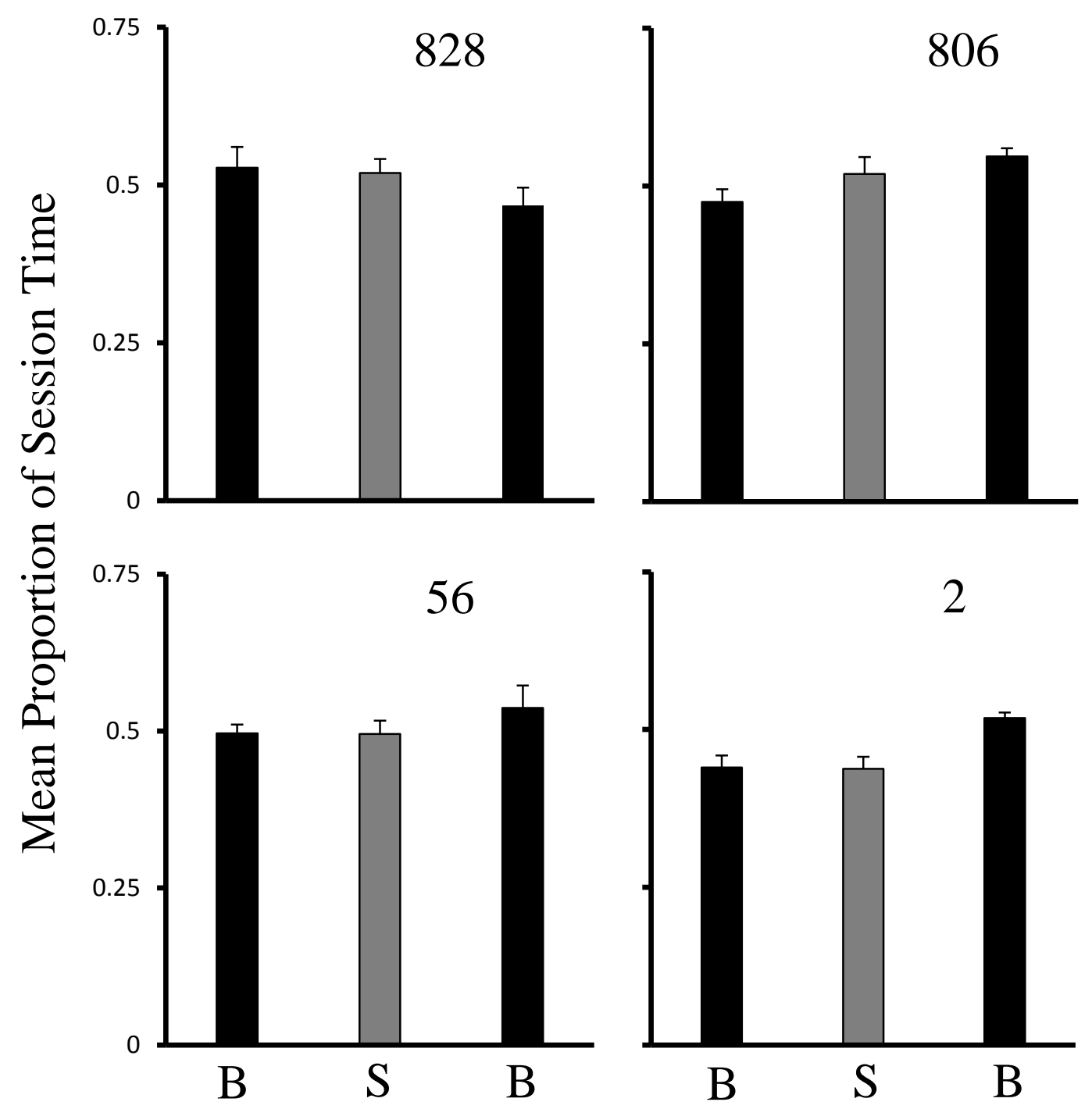

Figure 3. Mean proportions of session time calculated for one component from the last 6 stable sessions of each condition in Phase 2. $\mathrm{B}=$ Baseline, $\mathrm{S}=$ Signaled Onset. The baseline proportions correspond to the same component represented in the signaled onset proportion. Error bars represent the standard deviation of the mean. 

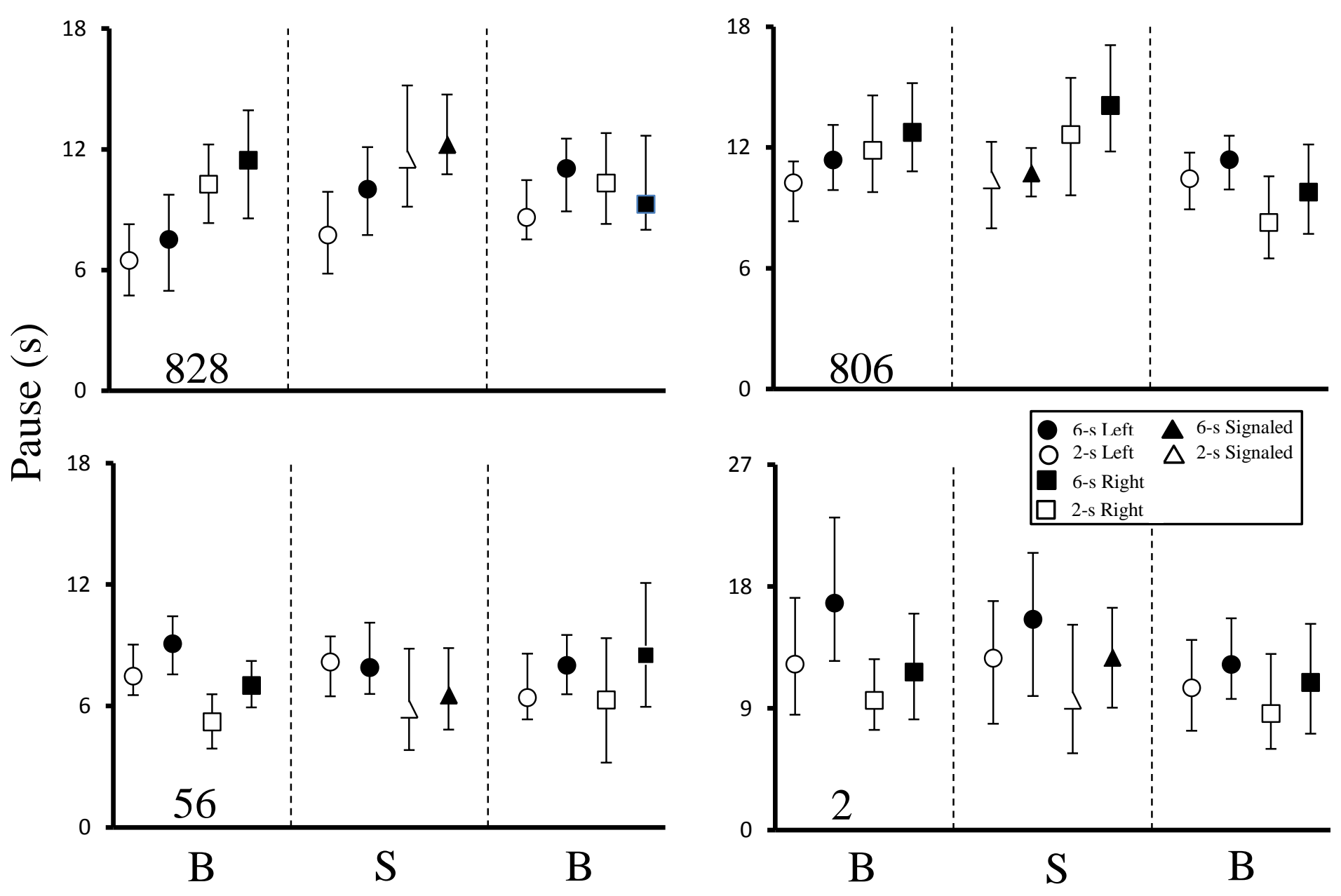

Figure 4. Latency to a changeover response following long and short reinforcer durations in both components for each condition of Phase 2. B = Baseline, $\mathrm{S}=$ Signaled Onset. Within each condition, the leftmost pair corresponds to the left hopper and the rightmost pair corresponds to the right hopper. Symbols represent medians and the bars represent the interquartile ranges (25th to 75th percentiles) of the last 6 stable sessions from each condition. 


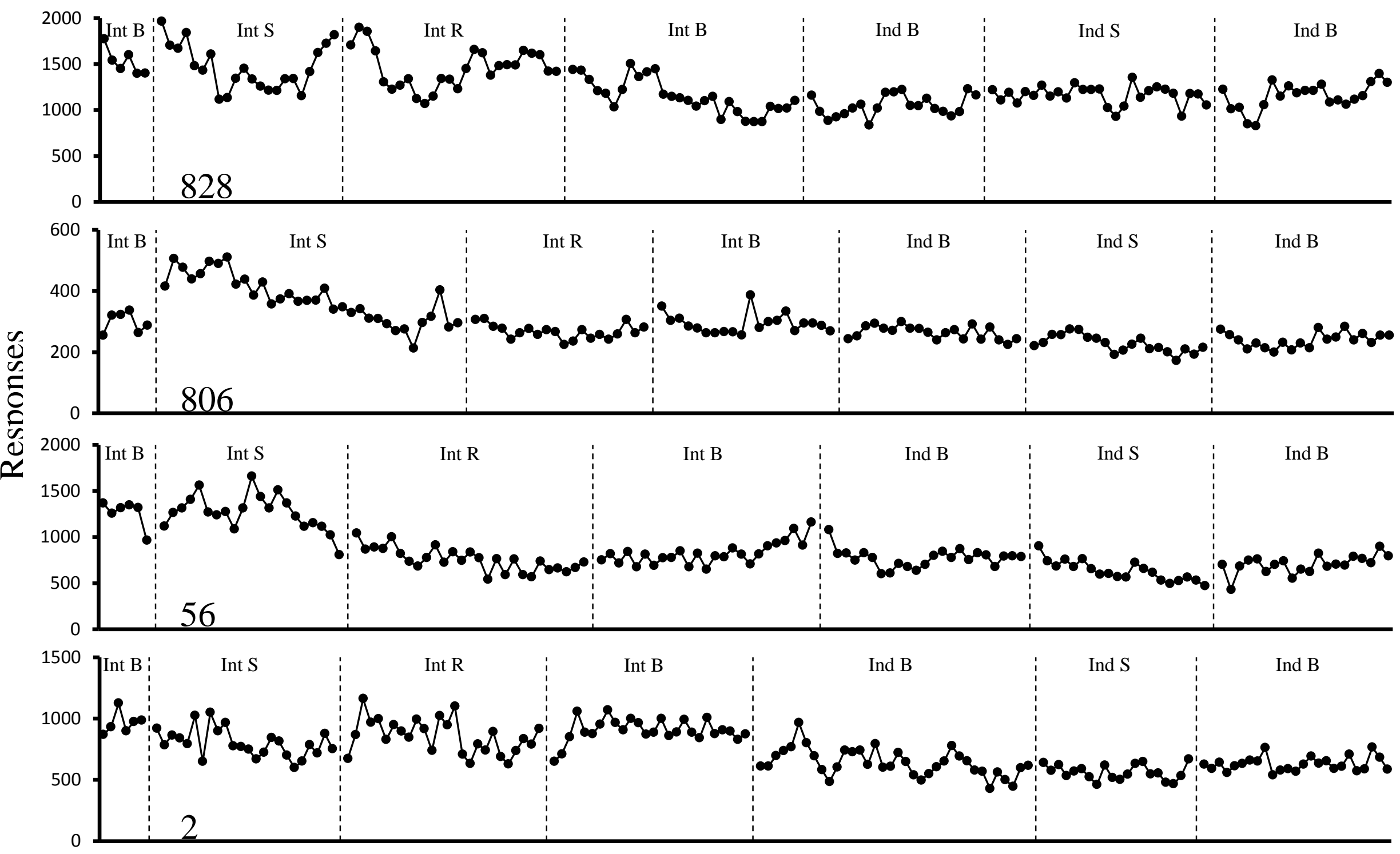

\section{Sessions}

Figure 5. Total changeover responses within each session for each condition of Phase 1 and Phase 2 starting with the last 6 stable sessions of the first baseline. Int = Interdependent, Ind = Independent, B = Baseline, S = Signaled Onset, R = Reversal. 
Table 5

Mean Reinforcement Rate in Hopper Presentations Per Minute for the Last 6 Stable Sessions of the Interdependent Concurrent VT 120 s VT 120 s Schedule

\begin{tabular}{lllll} 
Pigeon & Baseline 1 & Signaled Onset & Reversal & Baseline 2 \\
\hline \hline 828 & $0.86(0.02)$ & $0.88(0.01)$ & $0.89(0.02)$ & $0.90(0.02)$ \\
806 & $0.92(0.02)$ & $0.92(0.02)$ & $0.91(0.01)$ & $0.95(0.01)$ \\
56 & $0.90(0.02)$ & $0.90(0.01)$ & $0.90(0.01)$ & $0.88(0.02)$ \\
2 & $0.83(0.03)$ & $0.85(0.03)$ & $0.86(0.02)$ & $0.89(0.02)$ \\
\hline
\end{tabular}

Note. Values in parentheses are the standard deviation of the mean. 
Table 6

Mean Reinforcement Rate in Hopper Presentations Per Minute for the Last 6 Stable Sessions of the Independent Concurrent VT 120 s VT 120 s Schedule

\begin{tabular}{llll} 
Pigeon & Baseline 1 & Signaled Onset & Baseline 2 \\
\hline \hline 828 & $0.94(0.02)$ & $0.93(0.02)$ & $0.93(0.01)$ \\
806 & $0.95(0.01)$ & $0.95(0.01)$ & $0.95(0.01)$ \\
56 & $0.94(0.01)$ & $0.95(0.01)$ & $0.94(0.01)$ \\
2 & $0.93(0.02)$ & $0.95(0.01)$ & $0.93(0.02)$ \\
\hline
\end{tabular}

Note. Values in parentheses are the standard deviation of the mean. 


\section{Discussion}

The purpose of the present experiment was to examine whether enhancing the discrimination of reinforcer durations by signaling the onset of reinforcer magnitudes within one component of a concurrent schedule would result in differential control of time allocation in favor of the differentially signaled onsets. Time allocation, however, did not systematically vary as a function of signaling the onset of differential reinforcer durations presented in the signaled component. The latency to a changeover response did vary systematically as a function of the reinforcer duration: longer latencies occurred after the longer durations and shorter latencies occurred after the shorter durations.

The results of the experiment were consistent with previous studies examining reinforcement rate and reinforcer magnitude in concurrent schedules (Davison \& Hogsden, 1984). Consider the interdependent concurrent schedule of reinforcement (Stubbs \& Pliskoff, 1969) used in Phase 1. This schedule allowed a high degree of control over the distribution of reinforcers: a fixed relative rate of reinforcement set at 24 reinforcers for each component. The independent concurrent schedule used in Phase 2 relaxed this degree of control. Both schedules maintained a consistent overall session reinforcement rate, although the independent concurrent schedule rate was slightly higher than the interdependent concurrent schedule (see Tables 4 and 5 for a comparison of total session reinforcement rates). This finding demonstrates the reliability of the single-key concurrent schedule procedure in producing consistent reinforcement rates and time allocation between components for both schedule types.

The results of the latency to a changeover response analysis also are consistent with previous literature examining post reinforcement pauses following reinforcer magnitudes of different durations (Perone \& Courtney, 1992, Priddle-Higson, Lowe, \& Harzem, 1976, Lowe, 
Davey, \& Harzem, 1974). The current results extend the generality of these finding to VT concurrent schedules. Perone, Perone, and Baron (1987) suggest that pause duration is a function of the excitatory stimulus control exerted by the upcoming stimuli and magnitude correlation and the inhibitory aftereffects of reinforcement. They also suggested that these two variables often compete in controlling pausing. The current procedure did not allow discriminative control of behavior to develop between key color and reinforcer magnitude. There was no differential correlation of key light to reinforcer magnitude because the durations were equated within both components of the concurrent schedule and both reinforcer durations were assigned pseudo randomly to each component. This leaves inhibitory aftereffects as a plausible account for the differential pause durations shown in this study.

Mariner and Thomas (1969) suggested that the discriminability of different reinforcer durations could be enhanced by differentially signaling their onset. Similar results were not obtained with the current procedure. Methodological differences between the two experiments may account for these different outcomes. Mariner and Thomas used response rates as a measure of magnitude discrimination between components of a multiple schedule where 2 durations were strictly alternated. Greater differentiation in component rates for a signaled onset group compared to an unsignaled onset group was considered evidence of enhanced discrimination of reinforcer duration. Magnitude and stimulus conditions (key light color) covaried for both signaled onset and unsignaled onset groups and this produced results consistent with earlier findings of manipulating reinforcer magnitude between components of multiple schedules (Shettleworth \& Nevin, 1967). The effect of adding the differential onset stimuli may have enhanced magnitude discrimination, although, as previously mentioned the lack of stability 
criteria, limited number of sessions conducted, and the between-subject comparisons limit such conclusions based on their data.

The current procedure measured choice between signaled and unsignaled reinforcer onset within subject rather than response rate differences between subjects. The independent variable in the current experiment can be conceptualized as changing the stimuli accompanying the onset of different reinforcer durations in one component of a concurrent schedule from a mixed schedule onset (1 stimulus with 2 durations) to a multiple schedule onset (2 stimuli correlated with 2 durations). In Phase 1 of the experiment, reinforcer duration was equated in both components. This allowed the effects of signaling differential reinforcer onset to be isolated from changes in reinforcer magnitude, unlike in Mariner and Thomas (1969).

The effects of reinforcement rate, reinforcement magnitude or any interaction between the two may account for the inconsistent results of signaling reinforcer onset. Another possibility is that signaling the onset of differential reinforcer durations does not exert discriminative control over behavior relative to unsignaled onsets. Covarying stimuli correlated to different reinforcer magnitudes allows discriminative control of behavior to occur, but the procedures where such results are generally seen have not altered stimuli contiguous with reinforcer onset. The stimuli that generally covary are either distinct keys or levers (left or right) or combinations of other discriminative stimuli such as key light colors that occur before the onset of reinforcement. In this studies procedure, the most immediate effect of reinforcement was arranged to occur at the moment of onset. Because there was not a systematic increase in time allocation to the signaled onset component, it is not possible to make a complete determination about stimulus control by differential onset stimuli. Because Mariner and Thomas (1969) found differential effects of signaling reinforcer onset suggest, however, that the present 
negative results with respect to choice may be related to some feature of the procedure used in the analysis.

One such feature is that the potential reductions in overall and relative reinforcement rate that would occur as a result of overly biased time allocation to one alternative over the other in either concurrent schedule arrangement may have precluded any sustained shift in time allocation based on signaling differential reinforcer onsets. For example, if the subject allocated more time to one alternative there would necessarily be a decrease in the reinforcement rate obtained in that alternative. The results of the latency to a changeover response analysis revealed differential effects of magnitude that occurred before the onset stimuli were introduced and persisted after their introduction: longer latencies to changeover occurred following the larger reinforcer magnitudes. This was seen in both components of the concurrent schedules in both phases of the experiment. Therefore, signaling differential reinforcer onset was able to be isolated from changes in reinforcer magnitude between components, although the effect of reinforcer magnitude per se was not completely separable from the effects of introducing the reinforcer onset stimuli. This may have allowed the effect of reinforcer duration to overshadow any effects of reinforcer onset stimuli.

Overshadowing refers to a more "salient” stimulus reducing subsequent stimulus control of another stimulus (Pavlov, 1927; Mackintosh, 1974). An extended amount of exposure to both reinforcer durations occurred before the introduction of differentially signaled onset stimuli. The presence of and duration of access to the food may have been more discriminable than the hopper light. The differential stimulus control exerted by duration of access to reinforcement could have overshadowed development of stimulus control by the onset stimuli. Said another 
way, this might not have allowed discriminative control to develop to the onset stimuli (Dinsmoor, 1995).

Allowing exposure to differential onset stimuli to be under the control of the subject can potentially eliminate control by reinforcement rate and inhibitory aftereffects of reinforcement. For example, when observing responses produced stimuli correlated (not contiguous) with differential reinforcer durations, observing was maintained when such responses produced stimuli of sufficient duration and when they signaled larger reinforcer magnitudes (Auge, 1973). Another possibility is using a discrete trial procedure where "free choice" is given between signaled differential reinforcer durations and unsignaled durations. This would allow choice between a signaled and unsignaled alternative to occur independently from the effects of reinforcement rate and magnitude.

In Sidman's (1960) discussion of intrinsic versus imposed variability in Tactics of Scientific Research, he states that increasing the effectiveness of the maintaining variables within an experiment can not only result in decreased sensitivity of the behavior to extraneous variables, but also to the major variables under investigation. It is possible that behavior maintained under the concurrent schedule baseline in this experiment was not sensitive enough to examine the variable under investigation. If signaling the onset of differential reinforcer magnitudes is a relatively weak independent variable, then a high degree of control over the conditions in which the reinforcers are delivered may not be the best approach to study signaled onset. 


\section{References}

Auge, R.J. (1973). Effects of stimulus duration on observing behavior maintained by differential reinforcement magnitude. Journal of the Experimental Analysis of Behavior, 20, 429-438.

Baron, A., Mikorski, \& J., Schlung, M. (1992). Reinforcement magnitude and pausing on progressive-ratio schedules. Journal of the Experimental Analysis of Behavior, 58, 377388.

Baum, W. M., \& Rachlin, H. C. (1969). Choice as time allocation. Journal of the Experimental Analysis of Behavior, 12,861-874.

Bonem, M., \& Crossman, E.K. (1988). Elucidating the effects of reinforcement magnitude. Psychological Bulletin, 3, 148-362.

Brownstein, A. J. (1971). Concurrent schedules of response-independent reinforcement: Duration of a reinforcing stimulus. Journal of the Experimental Analysis of Behavior, 15, 211-214.

Brownstein, A. J., \& Pliskoff, S. S. (1968). Some effects of relative reinforcement rate and changeover delay in response-independent concurrent schedules of reinforcement. Journal of the Experimental Analysis of Behavior, 11, 683-688.

Catania, A.C. (1963). Concurrent performances: A baseline for the study of reinforcement magnitude. Journal of the Experimental Analysis of Behavior, 6, 299-300.

Davison, M., \& Hogsden, I. (1984). Concurrent variable-interval schedule performance: Fixed versus mixed reinforcer durations. Journal of the Experimental Analysis of Behavior, 41, 169-182.

Davison, M. (1988). Concurrent schedules: Interaction of reinforcer frequency and reinforcer duration. Journal of the Experimental Analysis of Behavior, 49, 339-349. 
Dinsmoor, J. A. (1995). Stimulus control: Part II. The Behavior Analyst, 18, 253-269.

Ettinger, R. H., McSweeney, F. K., \& Norman, W. D. (1981). Contrast and undermatching as a function of reinforcer duration and quality during multiple schedules. Journal of the Experimental Analysis of Behavior, 35, 271-281.

Findley, J.D. (1958). Preference and switching under concurrent scheduling. Journal of the Experimental Analysis of Behavior, 1, 123-144.

Fleshler, M., \& Hoffman, H. S. (1962). A progression for generating variable-interval schedules. Journal of the Experimental Analysis of Behavior, 5, 529-530.

Herrnstein, R.J. (1961). Relative and absolute strength of response as a function of frequency of reinforcement. Journal of the Experimental Analysis of Behavior, 4, 267-272.

Heyman, G. M., \& Monaghan, M. M. (1994). Reinforcer magnitude (sucrose concentration) and the matching law theory of response strength. Journal of the Experimental Analysis of Behavior, 61, 505-516.

Keesey, R. E., \& Kling, J. W. (1961). Amount of reinforcement and free-operant responding. Journal of Experimental Animal Behavior, 4, 125-132.

Lowe, C. F., Davey, G. C. L., \& Harzem, P. (1974). Effects of reinforcement magnitude on interval and ratio schedules. Journal of the Experimental Analysis of Behavior, 28, 553560.

Mackintosh, N. J. (1974). The Psychology of Animal Learning. Academic Press, London.

Mariner, R. W., \& Thomas, D. R. (1969). Reinforcement duration and the peak shift in postdiscrimination gradients. Journal of the Experimental Analysis of Behavior, 12, 759-766.

Neuringer, A. J. (1967). Effects of reinforcement magnitude on choice and rate of responding. Journal of the Experimental Analysis of Behavior, 10, 417-424. 
Pavlov, I. P. (1927). Conditioned Reflexes. Oxford University Press, Oxford.

Perone, M., \& Courtney, K. (1992). Fixed-ratio pausing: Joint effects of past reinforcer magnitude and stimuli correlated with upcoming magnitude. Journal of the Experimental Analysis of Behavior, 57, 33-46.

Perone, M., Perone, C. L., \& Baron, A. (1987). Inhibition by reinforcement: Effects of reinforcer magnitude and timeout on fixed-ratio pausing. The Psychological Record, 37, 227-238.

Ploog, B. O. (2001). Effects of primary reinforcement on pigeons' initial-link responding under a concurrent-chains schedule with nondifferential terminal links. Journal of the Experimental Analysis of Behavior, 76, 75-94.

Priddle-Higson, P. J., Lowe, C. F., \& Harzem, P. (1976). Aftereffects of reinforcement on variable-ratio schedules. Journal of the Experimental Analysis of Behavior, 25, 347-354.

Shettleworth, S., \& Nevin, J. A. (1965). Relative rate of response and relative magnitude of reinforcement in multiple schedules. Journal of the Experimental Analysis of Behavior, 8, 199-202.

Sidman, M. (1960). Tactics of Scientific Research. Basic Books, New York.

Stubbs, A.D., \& Pliskoff, S.S. (1969). Concurrent responding with fixed relative rate of reinforcement. Journal of the Experimental Analysis of Behavior, 12, 887-895.

Wyckoff, L. B. (1952). The role of observing responses in discrimination learning. Psychological Review, 59, 431-442.

Young, J. S. (1981). Discrete-trial choice in pigeons: Effects of reinforcer magnitude. Journal of the Experimental Analysis of Behavior, 3, 23-29. 